\title{
Polariton dispersion dependence on concentration of admixture in imperfect superlattice of coupled microresonators
}

\author{
A.P.Alodjants, V.V.Rumyantsev*, \\ S.A.Fedorov", M.V.Proskurenko* \\ Vladimir State University, 87 Gorky Str., 600000 Vladimir, Russia \\ *A.Galkin Physico-Technical Institute, National Academy of Sciences of \\ Ukraine, 72 R.Luxembourg Str., 83114 Donetsk, Ukraine
}

Received October 10, 2013

\begin{abstract}
Based on the representations of the ideal of photonic structures, the non-ideal systems of this class - polaritonic crystal, which is a set of spatially ordered cavities containing atomic clusters, is considered in the paper. Moreover, the spatial distribution of cavities (resonators) is translation invariant, and the atomic subsystem has randomly distributed defects: impurity atomic clusters (quantum dots) or a vacancies. Numerical modeling of dependence of the dispersion of polaritons in this imperfect superlattice of associated microresonators on impurity concentration is completed. Using the virtual crystal approximation the analytical expressions for polaritonic frequencies, effective mass and group velocities, as a function of corresponding quantum dots and vacancies concentrations, is obtained.
\end{abstract}

Рассмотрен поляритонный кристалл, представляющий собой совокупность пространственно упорядоченных ловушек-резонаторов, содержащих атомные кластеры. Причем пространственное распределение резонаторов - трансляционно инвариантно, а атомная подсистема имеет случайно распределенные дефекты: примесные атомные кластеры или вакансии. Выполнено численное моделирование зависимости дисперсии от концентрации примесей. В рамках приближения виртуального кристалла получены аналитические выражения для поляритонных частот, әффективной массы и групповой скорости в зависимости от концентрации дефектов.

Залежність дисперсії поляритонів від концентрації домішок у недосконалій надгратці пов'язаних мікрорезонаторів. А.П.Алоджанць, В.В.Рулянцев, С.А.Федоров, М.В.Проскуренко.

Розглянуто поляритонний кристал, що представляє собою сукупність просторововпорядкованих пасток-резонаторів, що містять атомні кластери. Просторовий розподіл резонаторів - трансляційно інваріантний, а атомна підсистема має випадково розподілені дефекти: домішкові атомні кластери (квантові точки) або вакансії. Виконано числене моделювання залежності дисперсії поляритонів у такій недосконалій надгратці пов'язаних мікрорезонаторів від концентрації домішок. у рамках наближення віртуального кристала отримано аналітичні вирази для поляритонних частот, ефективної маси і групової швидкості в залежності від концентрації домішкових квантових точок і вакансій.

\section{Introduction}

The important features of photonic bandgap structures under discussion [1] are con- nected with "slow" light, which is one of the promising fundamental physical phenomena that can be explored in the design 
of various quantum optical storage devices. In particular, the effective reduction of the group velocity demonstrated in the associated optical waveguide resonators [2, 3] as well as in the different types of solid-state semiconductor multilayer structures [4]. Key role in reducing the group velocity in these systems is played by so-called light and dark polaritons, which are linear superposition of photon states of the external electromagnetic field and the macroscopic (coherent) perturbations of two-level atomic medium.

In atomic systems, the lifetime of polaritons limited by lifetime of the excited atoms and is usually characterized by nanoscale [5]. The present level of development of nanotechnologies and nanophotonics makes it possible to study the "slow" light and the phase transitions of polaritons by creating a chain of coupled microcavities containing two-level atoms [6-8]. Technologically, the data structures can be obtained based on photonic crystals with defects as microcavities doped with two-level atoms [9].

In the context of this class of problems [10], a spatially periodic atomic structure - polaritonic crystal formed by ensembles of two-level atoms weakly interacting with the optical field in a tunnel connected array of microcavities is proposed in the paper. A remarkable feature of this structure is the possibility of localization of polaritons, which is similar to the possibility of localization of light in photonic crystals in nonlinear optics (see, eg, [9]) or the localization of excitons in quasi-periodic structures in solid state physics [11].

Based on the representations of the ideal photonic structures developed previously [12], the non-ideal system of this class the polaritonic crystal with the atomic subsystem containing the impurity atom clustersis considered in this paper. Moreover, the spatial distribution of traps-resonators remains, as before, translation invariant, but the atomic subsystem contains randomly distributed foreign (relative to the ideal system) quantum dots and/or vacancies. Each trap-cavity contains only one atomic complex of a certain type, or free from it (i.e, with a vacancy). Therefore, the parameters of the problem related to the atomic subsystem are configuration-dependent variables. Numerical modeling of such systems can be made under certain approximation, in particular the virtual crystal approximation (VCA) [13]. The last is to replace the configuration-dependent parame- ters of the Hamiltonian problem on a configuration-averaged their values. The analytical expressions for the polaritonic frequency, the effective mass and group velocity as a function of the impurity concentration of the quantum dots and vacancies is obtained in the paper in the framework of VCA. It turned out that even with a small number of vacancies in the lattice (one vacancy for a thousand cavities) mass of polaritons increases by three orders of magnitude.

\section{Polaritons in nonideal coupled microcavities lattice}

One way to create a polaritonic crystal is capture of two-level atoms in the photonic structure consisting of an array of tunnelcoupled microcavities (CROW) [2]. As in [6, 7], where the connected resonators doped with atoms in them, this study discussed 1D lattice microcavities containing one optical mode, each of which interacts with a neighbor in the chain. Thus, each resonator contains the macroscopic cluster of ultracold two level atoms of the same type with the levels $|a\rangle$ and $|b\rangle$ interacting with the quantized electromagnetic field directed (along the axis) perpendicular to the chain, which oriented along the $\mathrm{X}$ axis (see Fig. 1). In such a configuration the overlap of optical field and the wave functions of atoms is taken into account and there is a possibility photon tunneling along the chain resonators.

The hamiltonian $H$ of the system considered is:

$$
H=H_{a t}+H_{p h}+H_{i n t}
$$

Here $H_{a t}$ corresponds to an ensemble of two-level atoms (quantum dots) in the trapresonator, $H_{p h}$ corresponds to the propagation of the light field, $H_{\text {int }}$ describes the atom-optical interaction in the cavity.

Proceeding from the concepts developed, for example, in [10], the hamiltonian $H$ for

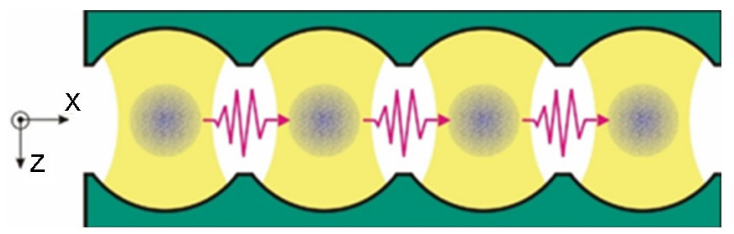

Fig. 1. A schematic model of polariton crystal: microresonators lattice containing macroscopic ensembles of two-level atoms and interacting with resonators electromagnetic modes. 
ideal photonic structures can be reduced to the form:

$$
\begin{gathered}
H_{a t}= \\
=\hbar \sum_{n=1}^{M}\left(\omega_{n, a t}^{(a)} a_{n}^{+} a_{n}+\omega_{n, a t}^{(b)} b_{n}^{+} b_{n}-\frac{\gamma_{a}}{2}\left(a_{n}^{+} a_{n-1}+a_{n}^{+} a_{n+1}+\text { H.C. }\right)-\right. \\
\left.-\frac{\gamma_{b}}{2}\left(b_{n}^{+} b_{n-1}+b_{n}^{+} b_{n+1}+\text { H.C. }\right)\right), \\
H_{p h}=\hbar \sum_{n=1}^{M}\left(\omega_{n, p h} \psi_{n}^{+} \psi_{n}-\frac{\alpha}{2}\left(\psi_{n}^{+} \psi_{n-1}+\psi_{n}^{+} \psi_{n+1}+H . C .\right)\right) \\
H_{\text {int }}=\hbar \sum_{n=1}^{M} \frac{g_{n}}{\sqrt{N}}\left(\psi_{n}^{+} a_{n}^{+} b_{n}+b_{n}^{+} a_{n} \psi_{n}\right),
\end{gathered}
$$

where the annihilation (creation) operators $a_{n}\left(a_{n}^{+}\right), \quad b_{n}\left(b_{n}^{+}\right)$in equation (2) characterize the dynamical properties of atomic ensembles (atomic quantum modes) at the lower $|a\rangle$ and upper $|b\rangle$ levels in the $n$-site of array $(n=1,2, \ldots M)$, and $\hbar \omega_{n, a t}^{(a)}$ and $\hbar \omega_{n, a t}^{(b)}$ characterize the energy of atom sat the levels, respectively. The coupling coefficients $\gamma_{a b}$ are the nearest-neighbor hopping constants that depend on overlapping in tegrals of the atomic cloud wave function. Wave functions (these functions are real Wannier functions) are responsible for spatial distribution of ultracold $n$-site atoms under the socalled strong-bonding approximation [14].

The annihilation (creation) operators $\psi_{n}\left(\psi_{n}^{+}\right)$ in (3) describe the temporal behaviour of a single photonic mode with frequency $\omega_{n, p h}$ located at the $n$-th cavity. The parameter $\alpha$ characterizes a spatial field overlapping between the neighboring cavities. The interaction of twolevel atoms with the quantized electromagnetic field in equation (4) is considered under the rotating wave approximation and determined by the constant $g_{n}$. It is assumed in framework of the polariton crystal model [12] that all the cells are identical to each other and have the same number of atoms $N_{n}=N$, as well as the coefficients of atomic-optical interaction in (4) are the same in all cells, that is $g=g_{1}=g_{2}=$ $\ldots=g_{M}$.

Let's consider the non-ideal system of this class - polaritonic structure, atomic subsystem that contains the impurity atom clusters. Moreover, the spatial distribution of traps resonators remains, as before, is translation invariant (with the lattice constant $l$ ), and the atomic subsystem contains randomly distributed for- eign (relative to the ideal system) quantum dots (each cavity contains only one atomic complex of a certain sort). Therefore, the parameters of the problem related to the atomic subsystem are configuration-dependent variables. Numerical simulation of such systems can be completed within the VCA $[15,16]$, which is to replace the configuration-dependent hamiltonian parameters $H_{a t}$ on their configuration averaged values. In this case, the configuration-dependent values are $\omega_{n, a t}^{(a, b)}, \gamma^{(a, b)}, g_{n}$, their configuration dependence is determined by the random variables:

$$
\begin{gathered}
\omega_{n, a t}^{(a, b)}=\sum_{v=1}^{r} \omega_{v, a t}^{(a, b)} \eta_{n}^{v}, \\
\gamma_{n, n \pm 1}^{(a, b)}=\sum_{v, \mu=1}^{r} \gamma_{v \mu}^{(a, b)} \eta_{n}^{v} \eta_{n \pm 1}^{\mu}, \\
g_{n}=\sum_{v}^{r}=1 g_{n}^{v v} \eta_{n}^{v} .
\end{gathered}
$$

Here configuration-dependent random unit $\eta_{n}^{v}$ equals 1 , if in node $n$ there is $v$-type, and it is zero in any other case (the number of atomic complex sorts are equal to $r$ ) and in any other case, besides $\sum_{v} \eta_{n}^{v}=1$.

After spending configuration averaging of these quantities we obtain:

$$
\begin{gathered}
\left\langle\omega_{n, a t}^{(a, b)}\right\rangle=\sum_{v} \omega_{v, a t}^{(a, b)} C^{v}, \\
\left\langle\gamma^{(a, b)}\right\rangle=\sum_{v, \mu} \gamma_{v \mu}^{(a, b)} C^{v} C^{\mu}, \\
\left\langle g_{n}\right\rangle=\sum_{v} g^{v} C^{v} .
\end{gathered}
$$

Here $C^{v}$ is the concentration of atomic clusters (quantum dots) type $v$, which satisfies the equality $\sum_{v} C^{v}=1 ; \omega_{n v, a t}^{(a, b)}, \gamma_{v \mu}^{(a, b)}, g^{\nu}$ are the characteristics telating to atomic components of -type. The procedure for configuration averaging (which indicated in (6) by angle brackets) allowed to "restor [17], the translational invariance of the hamiltonian and, therefore, to use the scheme of calculations [12].

We emphasize that the polaritonic model [12] atomic-optical interaction is valid only in the case of ultracold atoms with a "frozen" in a microcavity spatial degrees of freedom. Thus, the characteristic frequen- 
cies $\omega_{n, a t}^{(a, b)}, \omega_{n, p h}$, dependent on the potential traps in each cell. This approximation found to be valid when the number of atoms in the individual cells is relatively small $\left(N \leq 10^{4}\right)$ [18]. Parameter $g$ strong coupling of an atomoptical interaction satisfies the condition:

$$
g>>2 \pi / \tau_{c o h},
$$

i.e. $g$ in each cell of the lattice is much larger reverse atomic coherence time $\tau_{c o h}$ of optical system $[19,20]$. Physically, $\tau_{c o h}$ is the necessary time to achieve a thermal equilibrium for the atomic system under the interaction withthe quantized optical field in the polaritonic crystal (PolC) structure. In this case (at low temperatures of about $\mathrm{mK}$ ) spectral line broadening can be neglected. The result is a pure (thermodynamic equilibrium) quantum states of atomic-field systems.

Let us proceed to the k-representation in expressions (1) and (2). Taking into account the periodical properties of the system we can represent the operators $\psi_{n}, a_{n}, \psi_{n}$ in the form:

$$
\begin{gathered}
a_{n}=\frac{1}{\sqrt{M}} \sum_{k} a_{\boldsymbol{k}} e^{i \mathbf{k n}}, b_{n}=\frac{1}{\sqrt{M}} \sum_{k} b_{\boldsymbol{k}} e^{i \mathbf{k n},} \\
\psi_{n}=\frac{1}{\sqrt{M}} \sum_{k} \psi_{\boldsymbol{k}} e^{i \mathbf{k n},}
\end{gathered}
$$

where $\mathbf{n}$ is the lattice vector.

In the case of the investigated 1D PolC structure: $\mathbf{k n}=n k_{x} l, n=1,2, \ldots, M ; l$ is a lattice constant. Substituting equation (8) into equation (1) and taking into account the relation $\frac{1}{M} \sum_{n} e^{i\left(\mathbf{k}-\mathbf{k}^{\prime}\right) \mathbf{n}}=\delta_{\mathbf{k k}^{\prime}}$, we obtain the following expression for the hamiltonian (2):

$$
\begin{gathered}
H=\hbar \sum_{\mathbf{k}}\left(\omega_{p h}(k) \psi_{\mathbf{k}}^{+} \psi_{\mathbf{k}}+\right. \\
+\frac{\omega_{a t}(k)}{2}\left(b_{\mathbf{k}}^{+} b_{\mathbf{k}}\right)-\left(a_{\mathbf{k}}^{+} a_{\mathbf{k}}\right)+ \\
\left.+\frac{g}{\sqrt{N_{\text {tot }}}} \sum_{\mathbf{q}}\left(\psi_{\mathbf{k}}^{+} a_{\mathbf{q}}^{+} b_{\mathbf{k}+\mathbf{q}}+b_{\mathbf{k}+\mathbf{q}}^{+} a_{\mathbf{q}} \psi_{\mathbf{k}}\right)\right),
\end{gathered}
$$

where $N_{t o t}=N M$ is the total number of atoms for all sites; dispersion relations $\omega_{p h}(k)$ and $\left.\omega_{a t}\left(k,\left\{C^{v}\right)\right\}\right)$ for the photonic and atomic systems in the PolC structure defined by the following expressions:

$$
\omega_{p h}(k)=\omega_{n, p h}-2 \alpha \cos (k l),
$$

$$
\begin{gathered}
\omega_{a t}\left(k, C^{v}\right)=\sum_{v} C^{v}\left(\omega_{v, a t}^{(b)}-\omega_{v, a t}^{(a)}\right)- \\
-2 \sum_{v, \mu}\left(\gamma_{v, \mu}^{(b)}-\gamma_{v, \mu}^{(a)}\right) C^{v} C^{\mu} \cos k l,
\end{gathered}
$$

$\gamma=\gamma_{b}-\gamma_{a}$ is effective coupling coefficient of atomic clusters lattice.

With the help of the Bogolyubov transformations:

$$
\begin{gathered}
\Xi_{1, \mathbf{k}}=\vartheta_{1} \psi_{\mathbf{k}}-\vartheta_{2} P_{\mathbf{k}}, \\
\Xi_{2, \mathbf{k}}=\vartheta_{1} P_{\mathbf{k}}+\vartheta_{2} \psi_{\mathbf{k}}
\end{gathered}
$$

hamiltonian (9), which describes the light and dark polaritons [4], is reduced to a diagonal form:

$$
\begin{aligned}
H & =\hbar \sum_{\mathbf{k}} \Omega_{1}\left(k, C^{v}\right) \Xi_{1, \mathbf{k}}^{+} \Xi_{1, \mathbf{k}}+ \\
& +\hbar \sum_{\mathbf{k}} \Omega_{2}\left(k, C^{\vee}\right) \Xi_{2, \mathbf{k}}^{+} \Xi_{2, \mathbf{k}} .
\end{aligned}
$$

The annihilation operators $\Xi_{1, \mathrm{k}}, \Xi_{2, \mathrm{k}}$ in equation (12) characterize two types of quasi particles (due to the atom-field interaction) i.e. upper and lower branch polaritons, respectively. These quasi particles propagate along the $\mathrm{X}$-direction of the periodical structure. Hopfield coefficients $\vartheta_{1,2}$ satisfy to the normalization condition $\vartheta_{1}^{2}+\vartheta_{2}^{2}=1$.

We consider the limit of small perturbations, when all of the atoms in the cell are located on the lower level $|a\rangle$, i.e. $\sum_{\mathbf{q}}\left\langle b_{\mathbf{q}}^{+} b_{\mathbf{q}}\right\rangle<<\sum_{\mathbf{q}}\left\langle a_{\mathbf{q}}^{+} a_{\mathbf{q}}\right\rangle \approx N_{t o t}$, [19]. In this case, the operators $P_{\mathbf{k}}$ and $P_{\mathbf{k}}^{+}$of the atomic polarization and operators $\Xi_{1, \mathrm{k}}, \Xi_{2, \mathrm{k}}$, satisfy to the boson commutation relations:

$$
\begin{aligned}
{\left[P_{\mathbf{k}} ; P_{\mathbf{k}}^{+}=\right.} & \frac{1}{N_{t o t}} \sum\left(a_{\mathbf{q}}^{+} a_{\mathbf{q}}-b_{\mathbf{k}+\mathbf{q}}^{+} b_{\mathbf{k}+\mathbf{q}}\right) \approx 1, \\
& {\left[\Xi_{1, \mathbf{k}} ; \Xi_{1, \mathbf{q}}^{+}\right] \approx \delta_{i j} \delta_{\mathbf{k q}} }
\end{aligned}
$$

The characteristic frequencies $\Omega_{1,2}$ in (13) define an dispersion relations, as well as the polaritonic band structure of the crystal, they have a form:

$$
\begin{gathered}
\Omega_{1,2}\left(k, C^{\vee}\right)= \\
=\frac{1}{2}\left[\omega_{a t}\left(k, C^{\vee}\right)+\omega_{p h}(k) \pm \omega_{R}\left(k, C^{\vee}\right)\right],
\end{gathered}
$$


where $\omega_{R}\left(k, C^{v}\right)=\left[\delta^{2}=4\left(\sum_{v} g^{v} C^{v}\right)^{2}\right]_{\text {frequency, }}^{1 / 2}$ is the
Rabi $\delta=\omega_{p h}(k)-\omega_{a t}\left(k, C^{v}\right)$ is the phase mismatch depending on the quasi-momentum $k$. The expression for the effective mass $m_{1,2}\left(k,\left\{C^{\vee}\right)\right.$ of polaritons following from (15) is:

$$
\begin{gathered}
m_{1,2}\left(k, C^{v}\right) \equiv \hbar\left(\left.\frac{\partial^{2} \Omega_{1,2}\left(k, C^{v}\right)}{\partial k^{2}}\right|_{k=0}\right)^{-1}= \\
=\frac{2 m_{a t}\left(C^{v}\right) m_{p h}\left[\tilde{\Delta}^{2}\left(C^{v}\right)+4\left(\sum_{v} g^{v} C^{v}\right)^{2}\right]^{1 / 2}}{\left[m_{a t}\left(C^{v}\right)+m_{p h}\left[\tilde{\Delta}^{2}\left(C^{v}\right)+4\left(\sum_{v} g^{v} C^{v}\right)^{2}\right]^{1 / 2} \pm\left[m_{a t}\left(C^{v}\right)-m_{p h} \tilde{\Delta}\right.\right.} .
\end{gathered}
$$

Besides $\quad m_{p h}=\hbar / 2 \alpha l^{2}, \quad m_{a t}=$ $\hbar / 2 l^{2} \sum_{\nu, \mu}\left(\gamma_{v \mu}^{(b)}-\gamma_{\nu \mu}^{(a)}\right) C^{\nu} C^{\mu}$ and maximal detuning $\Delta$, which is independent on the frequency of the quasi-momentum is:

$$
\begin{aligned}
\tilde{\Delta} & =\omega_{p h}-\left[\sum_{v} C^{v}\left(\omega_{v, a t}^{(b)}-\omega_{v, a t}^{(a)}\right)\right]- \\
& -2\left[\alpha-\sum_{v, \mu}\left(\gamma_{v, \mu}^{(b)}-\gamma_{v, \mu}^{(a)}\right) C^{v} C^{\mu}\right] .
\end{aligned}
$$

The parameter $\alpha$ characterizes the spatial overlap between nearest resonators field [12]. The concentration dependence of the group velocities $V_{1,2}\left(k,\left\{C^{v}\right\}\right)$ is easily obtained by using expressions (15) and (16):

$$
\begin{gathered}
V_{1,2}\left(k, C^{\vee}\right) \equiv \frac{\partial \Omega_{1,2}\left(k, C^{\vee}\right)}{\partial k}= \\
=\frac{\hbar \sin (k l)}{2 l m_{p h}}\left[1+\frac{m_{p h}}{m_{a t}\left(\left\{C^{v}\right\}\right)} \pm\left(\frac{\left.1-m_{p h}\right)}{m_{a\{}\left(\left\{C^{v}\right\}\right)}\right) \frac{\omega_{p h}(k)-\omega_{a t}\left(k,\left\{C^{v}\right\}\right)}{\omega_{R}\left(k,\left\{C^{v}\right\}\right)}\right] .
\end{gathered}
$$

The concentration dependence of the obtained values enables to expand the range of possibilities of the numerical modeling and creating a new class of polariton crystal systems.

\section{Polaritonic crystal with the atomic subsystem containing vacancies}

To concretize the non-ideal polaritonic system, let's consider the case of the system containing only one type of atoms with the concentration $C_{1}$ and vacancies with concentration $C_{v}$, besides equality $C_{1}+C_{v}=1$ executes. At once variable $C_{1}$ is excluded from expressions using the equality $C_{1}=1-C_{v}$.
In this case, according to (15), we obtain:

$$
\begin{gathered}
\Omega\left(k, C_{v}\right) \equiv \Omega_{2}\left(k, C_{v}\right)= \\
=\frac{1}{2}\left[\omega_{a t}\left(k, C_{v}\right)+\omega_{p h}(k)-\omega_{R}\left(k, C_{v}\right)\right] .
\end{gathered}
$$

Here the dispersion relations for the atomic and photonic subsystems in a neighborhood $\operatorname{dot} k l=0$ defined as follows:

$$
\begin{gathered}
\omega_{a t}\left(k, C_{v}\right) \approx\left[\omega^{b a}+\gamma k^{2} l^{2}\right]-C_{v}\left[\omega^{b a}+\gamma k^{2} l^{2}\right] \\
\omega^{b a}=\omega_{n, a t}^{(b)}-\omega_{n, a t}^{(a)}-2 \gamma \\
\omega_{p h}(k) \approx \omega_{L}+\alpha k^{2} l^{2} \\
\omega_{L}=\omega_{n, p h}-2 \alpha
\end{gathered}
$$

and the Rabi frequency and atomic-optical detuning are:

$$
\begin{gathered}
\omega_{R}\left(k, C_{v}\right)=\sqrt{4 g^{2}\left(1-C_{v}\right)^{2}+\delta^{2}}, \\
\delta \equiv \delta\left(k, C_{v}\right)=\omega_{p h}(k)-\omega_{a t}\left(k, C_{v}\right) .
\end{gathered}
$$

In expression (20) appeared term $C_{v} \omega^{b a}$, which is extremely large in comparison, for example, with the average frequency of transition for rubidium D-line which is $\omega^{b a} \approx 2 \pi \cdot 382 \mathrm{THz}$. This term, even for a very small concentration of vacancies $\left(C_{v} \sim\right.$ $10^{-4}$ ) amounts to several THz. For comparison, the parameter $\alpha$ characterizing the photon tunneling, is from a few hundred $\mathrm{GHz}$ to $\mathrm{THz}$, and the parameter of atomicoptic communication $g$ consists of from tens to hundreds of $\mathrm{GHz}$. It are these parameters, $\alpha$ and $g$, even in the absence of vacancies are the main contributors to the features of the dispersion curve.

The expression for the mass of polaritons has the form:

$$
\begin{gathered}
m_{2}\left(C_{v}\right)= \\
=\frac{2 m_{a t}\left(C_{v}\right) m_{p h} \omega_{R}\left(C_{v}\right)}{\left.m_{a t}(C v)+m_{p h}\right) \omega_{R}\left(C_{n u)}-\left(m_{a t}\left(C_{v}\right)-m_{p h}\right) \Delta\left(C_{v}\right)\right.},
\end{gathered}
$$

where

$$
\begin{gathered}
\left.\omega_{R}\left(C_{\nu}\right) \equiv \omega_{R}\left(k, C_{v}\right)\right|_{k=0}= \\
=\sqrt{\left[\Delta\left(C_{v}\right)\right]^{2}+4 g^{2}\left(1-C_{v}\right)^{2}} .
\end{gathered}
$$

In formulas (23) and (24):

$$
\begin{gathered}
\left.\Delta\left(C_{v}\right) \equiv \delta\left(k, C_{v}\right)\right|_{k=0}=\Delta+C_{v} \omega^{b a}, \\
\left.\Delta \equiv \delta\left(k, C_{v}\right)\right|_{k=0_{C_{v}}}=\omega_{L}-\omega^{b a}, \\
m_{p h}=\hbar / 2 \alpha l^{2} \\
m_{a t}\left(C_{v}\right)=\hbar / 2 \gamma\left(1-C_{v}\right) l^{2}=M_{a t} /\left(1-C_{v}\right) .
\end{gathered}
$$


Fig. 2 shows the dependence of the mass $m_{2}$ of the polariton on concentration of vacancies $C_{v}$ introduced by the method described above. In this case, we used the following numerical values of the parameters: the size of the resonators is $l=3 \mu \mathrm{m}$, the effective mass of the atoms in the lattice without vacancies is $M_{a t}=1.44 \cdot 10^{-25} \mathrm{~kg}$, the effective mass of the photon is $m_{p h}=$ $2.8 \cdot 10^{-36} \mathrm{~kg}$, the atom-optical the detuning is $\Delta=0$ independent on the wave vector, the average frequency of the rubidium D-line is $\omega^{b a} \approx 2 \pi \cdot 382 \mathrm{THz}$, the parameter of atomic-optic coupling is $g=2 \pi \cdot 12.2 \mathrm{GHz}$. In the case of a lattice without vacancies the mass of polaritons is $m_{2} \approx 2 m_{p h} \approx 5.6 \cdot 10^{-36}$.

Analysis of the graph $m_{2}\left(C_{v}\right)$ shows that even for small number of vacancies $C_{v}$ in the lattice (one vacancy on $10^{-4}$ of resonators) the mass of polaritons increases by an order. The last circumstance testifies an essential role vacancies in effectively reducing of excitation velocity jumping between cavities.

\section{Conclusion}

Presented in the article study of the concentration dependence of the polariton parameters of imperfect 1D superlattice of coupled microcavities shows the significant role of admixtures included in the structure. In particular, the point defects in the PolC structure leads to increasing of the polariton effective mass, hence, to decreasing of the polariton group velocity (it is compared with the ideal polaritonic crystal $[12,16])$. This resume is illustrated by a specific example of the polaritonic crystal with the atomic subsystem containing vacancies. The results of the numerical simulation allow to expand opportunities of creatinga new class of functional materials - polaritonic crystal systems.

The work was performed as part of a joint project of the National Academy of Sciences of Ukraine and the Russian Foundation for basic research No.0112U004002, as well as the European project FP7-PEOPLE2013-IRSES No.612600 "LIMACONA".

\section{References}

1. P.W.Milonni, Fast Light, Slow Light and Left-Handed Light, Institute of Physics Publishing, Bristol (2005).

2. Z.S.Yang, N.H.Kwong, R.Binder, A.L.Smirl, J.Opt.Soc.Am. B, 22, 2144 (2005).

3. H.Gersen, T.J.Karle, R.J.P.Engelen et al., Phys. Rev.Lett., 94, 073903 (2005).

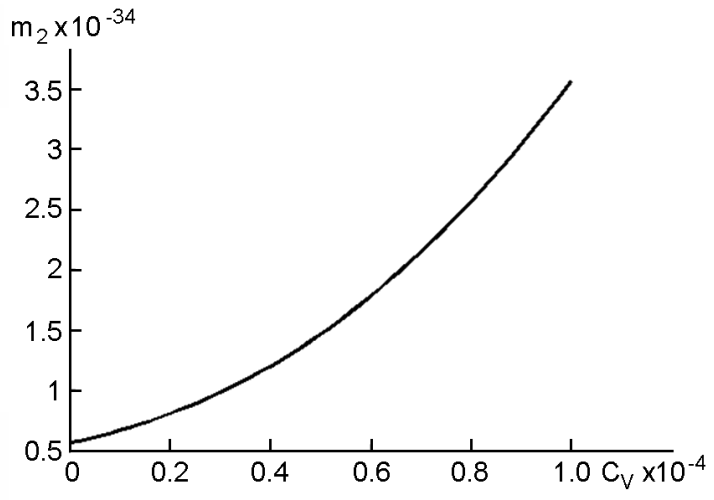

Fig. 2. The dependence of polaritonic mass on vacancies in the imperfect superlattice of coupled microresonators.

4. A.V.Turukhin, V.S.Sudarshanam, M.S.Shahriar et al., Phys.Rev.Lett., 88, 023602-1 (2002).

5. U.Vogl, M.Weitz, Phys.Rev.A, 78, 011401 (2008).

6. T.Aoki, B.Dayan, E.Wilcut et al., Nature, 443, 671 (2006).

7. M.J.Hartmann, F.Brandao, M.B.Plenio, $\mathrm{Na}$ ture, 2, 849 (2006).

8. L.Zhou, J.Lu, C.P.Sun, Phys.Rev.A, 76, 012313 (2007).

9. J.D.Joannopoulos, S.G.Johnson, J.N.Winn et al., Photonic Crystals. Molding the Flow of Light, 2-nd Edition, Princeton University Press, Princeton (2008).

10. E.S.Sedov, A.P.Alodjants, S.M.Arakelian et al., Phys. Rev.A, 84, 013813 (2011).

11. E.L.Albuquerque, M.G.Cottam, Polaritons in Periodic and Quasi Periodic Structures, Elsevier, Amsterdam (2004).

12. A.P.Alodjants, I.O.Barinov, S.M.Arakelian, Phys. B, 43, 095502 (2010).

13. R.H.Parmenter, Phys. Rev., 97, 587 (1955).

14. A.Smerzi, A.Trombettoni, P.G.Kevrekidis, A.R.Bishop, Phys. Rev. Lett., 89, 170402-1 (2002).

15. V.V.Rumyantsev, S.A.Fedorov, K.V.Gumennyk, Photonic Crystals: Optical Properties, Fabrication and Applications, ed. by William L.Dahl, Nova Science Publishers, Inc. NY: (2011).

16. V.V.Rumyantsev, A.P.Alodjants, S.A.Fedorov, Photonic and Electronic Excitations in Nonideal Superlattices, LAP LAMBERT Academic Publishing, Saarbrucken, Germany: (2013).

17. J.M.Ziman, Models of Disorder, John Willey \& Sons, New York (1979).

18. J.R.Anglin, A.Vardi, Phys. Rev.A, 64, 013605 (2001).

19. A.P.Alodjants, S.M.Arakelian, A.Yu.Leksin, Laser Phys., 17, 1432 (2007).

20. V.A.Averchenko, A.P.Alodzhants, S.M. Arakelyan et al., Quant. Electron., 36, 532 (2006). 